\title{
Genetically determined telomere length and multiple myeloma risk and outcome
}

Matteo Giaccherini (10), Angelica Macauda², Enrico Orciuolo³, Marcin Rymko4, Karolina Gruenpeter ${ }^{5}$, Charles Dumontet ${ }^{6}$, Malgorzata Raźny ${ }^{7}$, Victor Moreno $\mathbb{0}^{8}$, Gabriele Buda ${ }^{3}$, Katia Beider ${ }^{9}$, Judit Varkonyi ${ }^{10}$, Hervé Avet-Loiseau ${ }^{11}$, Joaquín Martinez-Lopez $\mathbb{1}^{12}$, Herlander Marques ${ }^{13}$, Marzena Watek ${ }^{14,15}$, Maria Eugenia Sarasquete $\mathbb{1}^{16}$, Vibeke Andersen ${ }^{17,18}$, Lionel Karlin ${ }^{6}$, Anna Suska ${ }^{19}$, Marcin Kruszewski ${ }^{20}$, Niels Abildgaard ${ }^{21}$, Marek Dudziński ${ }^{22}$, Aleksandra Butrym ${ }^{23}$, Arnold Nagler ${ }^{9}$, Annette Juul Vangsted $\mathbb{0}^{24}$, Katalin Kadar ${ }^{10}$, Tomczak Waldemar $\mathbb{1}^{25}$, Krzysztof Jamroziak ${ }^{15}$, Svend Erik Hove Jacobsen ${ }^{17}$, Lene Hyldahl Ebbesen ${ }^{26}$,

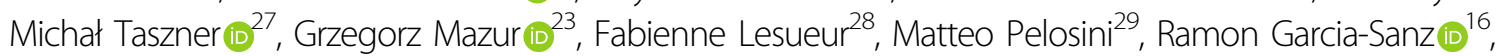
Artur Jurczyszyn (iD ${ }^{19}$, Delphine Demangel ${ }^{6}$, Rui Manuel Reis ${ }^{13,30}$, Elżbieta Iskierka-Jażdżewska ${ }^{31}$, Miroslaw Markiewicz $\mathbb{D}^{22}$, Federica Gemignani ${ }^{1}$, Edyta Subocz ${ }^{32}$, Daria Zawirska ${ }^{33}$, Agnieszka Druzd-Sitek ${ }^{34}$, Anna Stępieńn ${ }^{35}$, M. Henar Alonso ${ }^{8}$, Juan Sainz $\mathbb{0}^{36,37}$, Federico Canzian $\mathbb{1}^{2}$ and Daniele Campa ${ }^{1}$

\begin{abstract}
Telomeres are involved in processes like cellular growth, chromosomal stability, and proper segregation to daughter cells. Telomere length measured in leukocytes (LTL) has been investigated in different cancer types, including multiple myeloma (MM). However, LTL measurement is prone to heterogeneity due to sample handling and study design (retrospective vs. prospective). LTL is genetically determined; genome-wide association studies identified 11 SNPs that, combined in a score, can be used as a genetic instrument to measure LTL and evaluate its association with MM risk. This approach has been already successfully attempted in various cancer types but never in MM. We tested the "teloscore" in 2407 MM patients and 1741 controls from the International Multiple Myeloma rESEarch (IMMeNSE) consortium. We observed an increased risk for longer genetically determined telomere length (gdTL) $(O R=1.69 ; 95 \%$ Cl 1.36-2.11; $P=2.97 \times 10^{-6}$ for highest vs. lowest quintile of the score). Furthermore, in a subset of $1376 \mathrm{MM}$ patients we tested the relationship between the teloscore and $\mathrm{MM}$ patients survival, observing a better prognosis for longer gdTL compared with shorter gdTL ( $H R=0.93 ; 95 \% \mathrm{Cl} 0.86-0.99 ; P=0.049)$. In conclusion, we report convincing evidence that longer gdTL is a risk marker for MM risk, and that it is potentially involved in increasing MM survival.
\end{abstract}

\section{Introduction}

Multiple myeloma (MM) is a plasma cell malignancy that arises from a single clone of malignant plasma cells (PCs) in the bone marrow. It has a worldwide incidence of $2.1 / 100,000$ new cases every year, ranking as the second

\footnotetext{
Correspondence: Federico Canzian (f.canzian@dkfz.de)

${ }^{1}$ Department of Biology, University of Pisa, Pisa, Italy

${ }^{2}$ Genomic Epidemiology Group, German Cancer Research Center (DKFZ),

Heidelberg, Germany

Full list of author information is available at the end of the article

These authors contributed equally: Federico Canzian, Daniele Campa
}

most common hematological cancer ${ }^{1}$. MM has usually a late onset with a mean age at diagnosis around 60 years ${ }^{1}$.

There are overwhelming evidences that genetic variability influences the risk of developing $\mathrm{MM}^{2-8}$. In the recent years the importance of genetics has also emerged in MM response to treatment and survival ${ }^{9-15}$. Alongside polymorphic variants, another emerging marker of susceptibility and prognosis for several diseases is telomere length. Telomeres are specialized structures that cap the end of chromosomes and are involved in several processes like cellular growth, chromosomal stability, and proper segregation to

\section{(c) The Author(s) 2021}

(c) (i) Open Access This article is licensed under a Creative Commons Attribution 4.0 International License, which permits use, sharing, adaptation, distribution and reproduction cc) in any medium or format, as long as you give appropriate credit to the original author(s) and the source, provide a link to the Creative Commons license, and indicate if changes were made. The images or other third party material in this article are included in the article's Creative Commons license, unless indicated otherwise in a credit line to the material. If material is not included in the article's Creative Commons license and your intended use is not permitted by statutory regulation or exceeds the permitted use, you will need to obtain permission directly from the copyright holder. To view a copy of this license, visit http://creativecommons.org/licenses/by/4.0/. 
daughter cells ${ }^{16}$. Telomerases are the most important enzymes involved in telomere replication and their dysfunction is considered a cancer hallmark ${ }^{17}$. Telomere length measured in leukocytes (LTL) has been used to investigate the susceptibility to different cancer types including several hematological malignancies ${ }^{18-25}$, among which MM for which only two studies of small size have been attempted, a prospective cohort study and a retrospective case control study $^{21,26}$. In a meta-analysis conducted by $\mathrm{Xu}$ and colleagues the role of LTL in relation to survival in several cancer types has been investigated ${ }^{27}$. However, MM was not among the tumors investigated by the authors and the only study conducted on MM survival consisted of five patients where telomere length was measured in bone marrow cells, comparing malignant vs. non-malignant cells ${ }^{28}$.

Telomere length is highly correlated across tissues ${ }^{29,30}$, it is reasonably stable during time, as assessed in a longitudinal study $^{18}$, and therefore it is considered a valid surrogate for the measure of telomere length in specific tissues.

However, LTL measurement is prone to heterogeneity due to sample handling (DNA extraction methods used, storage of blood and/or DNA, elution buffer used) ${ }^{31}$. In addition, the analysis of LTL is sensitive to other confounders, such as the epidemiologic design of the study (retrospective vs. prospective) and, in studies on cancer, whether the subjects have received chemotherapy or not. LTL is genetically determined (gdTL) with an estimated heritability that ranges from 0.44 to $0.70^{32,33}$. Recently genome-wide association studies (GWAS) have identified $11 \mathrm{SNPs}^{23,34-36}$ that, collectively, explain $2.28 \%$ of LTL variability and can be used as a genetic instrument in a Mendelian randomization fashion. This approach has been already successfully tested in relation to the risk of developing various cancer types ${ }^{23,37-45}$, but was never done in MM. We have tested the genetic score (henceforth called "teloscore") in the context of the International Multiple Myeloma rESEarch (IMMeNSE) consortium to investigate the relationship between gdTL and $\mathrm{MM}$ risk and survival.

\section{Materials and methods}

\section{Study population}

This study was conducted within the IMMeNSE consortium, that has been extensively described elsewhere ${ }^{46}$. We used DNA samples from 2407 MM patients and 1741 controls for whom information on sex, age (age at diagnosis for the cases/age at recruitment for the controls), and country of origin (Denmark, France, Hungary, Israel, Italy, Poland, Portugal, and Spain) was collected. Cases were defined by a confirmed diagnosis of MM according to the International Myeloma Working Group (IMWG) criteria $^{47}$. In addition, clinical data such as disease stage according to Durie-Salmon (DS) staging system ${ }^{48}$ and/or International Staging System (ISS) ${ }^{49}$, first-line treatment received, based on bortezomib/immunomodulatory drugs (defined as "new treatments") or any other regimen, such as vincristine/adriamycin/dexamethasone or melphalan/ prednisone (defined as "old treatments") and overall survival (OS) were retrospectively collected from medical records. Information about DS stage was available for 1235 cases, while the stage according to ISS was available for 1022 cases, and for 984 cases information on both systems was available. Controls were selected among the general population as well as among hospitalized subjects or among blood donors in the same geographic areas where the cases were collected. Characteristics of the study population are summarized in Table 1 . The

Table 1 Summary of the study population.

\begin{tabular}{|c|c|c|}
\hline & MM cases & Controls \\
\hline \multicolumn{3}{|l|}{ Country/region } \\
\hline Denmark & 277 & 494 \\
\hline France & 330 & 176 \\
\hline Hungary & 83 & 75 \\
\hline Israel & 89 & 72 \\
\hline Italy & 231 & 227 \\
\hline Poland & 1043 & 262 \\
\hline Portugal & 126 & 120 \\
\hline Spain & 228 & 315 \\
\hline Total & 2407 & 1741 \\
\hline \multicolumn{3}{|l|}{ Sex } \\
\hline Male & $53.26 \%$ & $51.92 \%$ \\
\hline Female & $46.74 \%$ & $48.08 \%$ \\
\hline Median age & 61.47 & 52.59 \\
\hline (25th - 75th percentile) & $(55-68)$ & $(42-65)$ \\
\hline \multicolumn{3}{|c|}{ Disease stage, Durie-Salmon } \\
\hline 1 & 179 & \\
\hline 2 & 299 & \\
\hline 3 & 757 & \\
\hline Total & 1235 & \\
\hline \multicolumn{3}{|c|}{ Disease stage, International Staging System } \\
\hline 1 & 324 & \\
\hline 2 & 332 & \\
\hline 3 & 366 & \\
\hline Total & 1022 & \\
\hline \multicolumn{3}{|l|}{ Vital status } \\
\hline Alive & 902 & \\
\hline Deceased & 371 & \\
\hline Total & 1273 & \\
\hline
\end{tabular}


IMMEnSE study protocol was approved by the Ethics Committee of the Medical Faculty of the University of Heidelberg (reference number: S-004/2020). Following the guidelines of the Declaration of Helsinki, written informed consent was obtained from each participant.

\section{SNP selection}

To build the teloscore we used 11 single nucleotide polymorphisms (SNPs) (ZNF676-rs412658, TERT-rs2736100, CTC1-rs3027234, DHX35-rs6028466, PXK-rs6772228, NAF1-rs7675998, ZNF208-rs8105767, OBFC1-rs9420907, ACYP2-rs11125529, TERC-rs10936599, and ZBTB46rs755017), identified through GWAS, that affect telomere length ${ }^{23,34-36}$. Characteristics of the SNPs under investigation are summarized in Supplementary Table 1.

\section{Genotyping}

DNA samples were extracted from whole blood. Genotyping was conducted in 384-well plates, using TaqMan (ABI, Applied Biosystems, Foster City, CA, USA) technology according to the manufacturer's recommendations. For quality control purposes around $10 \%$ of the samples were duplicated in order to check the concordance between the genotypes. Case/control status was unknown to the person performing the genotyping. Fluorescent signals of the genotyping assays were read on PCR plates by a spectrophotometer $(\Omega$, BMG LABTECH, Ortenberg, Germany) and the software KlusterCaller (LGC Group, Teddington, UK) was used to determine genotypes.

\section{Teloscore computation}

The "teloscore" was computed as follows: for each SNP the number of alleles associated with longer telomeres (according to the results of the literature) was counted and added up for each study subject, resulting in the unweighted score. Therefore, considering 11 SNPs, the unweighted score could assume any value between 0 (shortest telomeres) and 22 (longest telomeres). We then created a weighted score for each study subject. First, we took from the literature estimates of the per-allele effect on LTL in base pairs for each SNP (Supplementary Table 1). Then, we multiplied for each SNP the number of alleles associated with longer telomeres by the per-allele effect on LTL in base pairs that were then summed up for each study subject. The weighted score thus represents the estimated difference in telomere length, measured in base pairs, attributable to the SNPs under investigation. The concordance between the teloscore built with SNPs and the relative telomere length measured with real time quantitative PCR was described in detail elsewhere ${ }^{37}$. Only a subset of the study subjects had a 100\% SNP call rate (1769 cases and 1207 controls). For survival analyses, unweighted and weighted teloscores were built with all the individuals with complete data on stage, therapy received and OS. The number of cases with $100 \%$ SNP call rate was 1000 with DS staging system information and 813 with ISS.

In order to be able to compute comparable score values for all study subjects, we also considered average values for each score. Supplementary Table 2 shows examples of how the teloscores were generated.

\section{Statistical analysis}

The association between the individual SNPs and MM risk was tested using unconditional logistic regression computing odds ratios (ORs) and 95\% confidence intervals (CIs). We used an allelic (log-additive) and a codominant model of inheritance. The threshold for statistical significance was therefore $p=0.05 /(11$ SNPs $\times 2$ models $)$ $=0.05 / 22=0.0023$.

For both teloscores (weighted and unweighted) we calculated quintiles based on the distribution of values in the controls. The association between the teloscores and MM risk was tested with logistic regression computing OR and 95\% CIs.

All analyses were adjusted for age, sex, and country of origin.

Survival analysis to assess the effect of the SNPs and the teloscore on OS of MM patients was done using Cox regression, calculating hazard ratio (HR), and $95 \%$ CI. OS was defined as the time between MM diagnosis and last follow up for alive patient or death. Survival analyses were carried out with individual SNPs and with both teloscores (weighted and unweighted). We built the teloscores calculating quintiles based on the distribution of values in all the individuals with staging, therapies and OS data. The analyses were adjusted for age, sex, country of origin, MM stage (according to DS or ISS), and first-line therapy.

To better assess the causality of the association between LTL and MM onset and progression we used a Mendelian randomization approach considering the 11 SNPs as genetic instruments of the exposure, and risk of developing MM and MM OS as outcomes of interest. We utilized the inverse variance weighted (IVW) regression because on the premise of absence of directional pleiotropy, it provides consistently robust causal effect estimates ${ }^{50,51}$. The Egger (MR-Egger) estimation method was used to test for possible pleiotropic effects of the genetic instruments ${ }^{50,51}$. Finally to assess evidence of heterogeneity between the polymorphisms, the Cochran $\mathrm{Q}^{\prime}$ heterogeneity and Bowden $\mathrm{I}_{\text {GX }}^{2}$ statistics were computed to obtain an estimate of instrument strength for the MR-Egger analysis ${ }^{52}$. Additional information has been given elsewhere ${ }^{50,51}$. 
Analyses were carried out with the Mendelian randomization package in R V0.5.0 (30 Sept. 2020).

\section{Bioinformatic tools}

Several bioinformatic tools were used in order to identify functional relevance for the polymorphic variants showing the most promising associations with risk of developing MM. In particular, RegulomeDB (https:// www.regulomedb.org/regulome-search/ $)^{53}$ was used to identify the regulatory potential of the region nearby each SNP while the GTEx portal web site (https://www. gtexportal.org) was used to identify potential associations between the SNP and expression levels of nearby genes $(\mathrm{eQTL})^{54}$.

\section{Results}

Characteristics of the study population are summarized in Table 1. The SNPs under investigation are summarized in Supplementary Table 1. All the selected SNPs were in Hardy-Weinberg Equilibrium (HWE) in the control population. The average call rate of the SNPs used for statistical analysis was $96.3 \%$. Concordance with duplicated samples was higher than $99 \%$.

We observed several associations between individual SNPs and MM risk. In particular, two polymorphisms showed a statistically significant association considering the Bonferroni-corrected threshold $(p<0.0023)$. TERCrs10936599-T was associated with decreased risk of developing MM $\left(\mathrm{OR}_{\text {homozygous }}=0.55 ; 95 \%\right.$ CI $0.39-0.76 ; P=$ $2.87 \times 10^{-4}$ ), while $O B F C 1$-rs9420907-C was associated with increased $\mathrm{MM}$ risk $\left(\mathrm{OR}_{\text {heterozygous }}=1.32 ; 95 \% \mathrm{CI} 1.12-1.55\right.$;
$\left.P=7.78 \times 10^{-4}\right)$. All the results of the risk analysis considering the SNPs individually are shown in Table 2.

\section{Association of the "teloscore" with MM risk}

Supplementary Table 2 illustrates how the teloscores were generated. We tested the association between the unweighted and weighted teloscore with MM risk in individuals that had $100 \%$ call rate only (1769 cases and 1207 controls). We observed an increased risk for individuals that had longer gdTL. The association was similar in terms of estimates and $P$ values when comparing individuals belonging to the highest quintile with individuals belonging to the lowest quintile, for the unweighted $(\mathrm{OR}=$ 1.36; $95 \%$ CI 1.07-1.73; $P=0.013)$ and weighted $(\mathrm{OR}=$ 1.39; 95\% CI 1.08-1.80; $P=0.011$ ) scores (Table 3).

We performed an additional analysis using all the individuals and the average scores rather than the absolute values (see Methods). We observed a strong association between long gdTL and increased risk of MM when analyzing the score as a categorical variable with a stronger association observed with the weighted score $\left(\mathrm{OR}=1.69 ; 95 \% \mathrm{CI} 1.36-2.11 ; P=2.97 \times 10^{-6}\right.$ for highest vs. lowest quintile) compared to the unweighted score $\left(\mathrm{OR}=1.58\right.$; 95\% CI 1.28-1.94; $P=1.93 \times 10^{-5}$ ) (Table 3).

Considering that TERC-rs10936599 is a well-known risk locus for MM, we have also computed a score considering all the SNPs with the exception of this one, observing results that are in line with what we observed with the 11 SNP score (Supplementary Table 3).

We also estimated the correlation between MM risk and the genetic score using a Mendelian randomization

Table 2 Association between single SNPs and MM risk.

\begin{tabular}{|c|c|c|c|c|c|c|c|c|c|c|c|c|c|}
\hline \multirow[t]{2}{*}{ SNP } & \multirow[t]{2}{*}{ Gene } & \multirow{2}{*}{$\begin{array}{l}\text { Allele } \\
\mathrm{M} / \mathrm{m}\end{array}$} & \multirow[t]{2}{*}{$\mathrm{MAF}^{\mathrm{a}}$} & \multirow[t]{2}{*}{$E A^{a}$} & \multicolumn{3}{|c|}{ Allelic model $^{\mathbf{b}}$} & \multicolumn{6}{|c|}{ Codominant model $^{c}$} \\
\hline & & & & & $\mathrm{HR}^{1}$ & $95 \% \mathrm{Cl}^{1}$ & $P_{\text {value }}$ & $\mathrm{HR}_{\mathrm{Het}}$ & $95 \% \mathrm{Cl}$ & $P_{\text {value }}$ & $\mathrm{HR}_{\mathrm{Hom}}$ & $95 \% \mathrm{Cl}$ & $P_{\text {value }}$ \\
\hline rs11125529 & ACYP2 & $C / A$ & 0.11 & $A$ & 0.99 & $0.86-1.16$ & 0.994 & 1.10 & $0.92-1.31$ & 0.299 & 0.63 & $0.37-1.07$ & 0.093 \\
\hline rs6772228 & PXK & T/A & 0.04 & $\mathrm{~T}$ & 0.81 & $0.62-1.07$ & 0.121 & 0.75 & $0.50-0.99$ & 0.048 & 1.91 & $0.43-8.32$ & 0.389 \\
\hline rs10936599 & TERC & $C / T$ & 0.24 & $C$ & 0.79 & $0.70-0.90$ & $1.72 \times 10^{-4}$ & 0.84 & $0.72-0.98$ & 0.027 & 0.55 & $0.39-0.76$ & $2.87 \times 10^{-4}$ \\
\hline rs7675998 & NAF1 & $\mathrm{G} / \mathrm{A}$ & 0.24 & G & 0.94 & $0.83-1.06$ & 0.321 & 0.84 & $0.73-0.98$ & 0.029 & 1.15 & $0.82-1.60$ & 0.402 \\
\hline rs2736100 & TERT & $\mathrm{A} / \mathrm{C}$ & 0.5 & $C$ & 1.07 & $0.96-1.18$ & 0.223 & 1.07 & $0.89-1.28$ & 0.479 & 1.14 & $0.92-1.39$ & 0.223 \\
\hline rs9420907 & $O B F C 1$ & $\mathrm{~A} / \mathrm{C}$ & 0.13 & $C$ & 1.17 & $1.03-1.35$ & 0.020 & 1.32 & $1.12-1.55$ & $7.78 \times 10^{-4}$ & 0.88 & $0.57-1.35$ & 0.556 \\
\hline rs3027234 & CTC1 & $C / T$ & 0.22 & $C$ & 0.89 & $0.79-1.00$ & 0.054 & 0.97 & $0.83-1.13$ & 0.669 & 0.65 & $0.47-0.90$ & 0.009 \\
\hline rs8105767 & ZNF208 & $\mathrm{A} / \mathrm{G}$ & 0.28 & G & 1.14 & $1.02-1.27$ & 0.022 & 1.05 & $0.90-1.21$ & 0.559 & 1.46 & $1.12-1.90$ & 0.005 \\
\hline rs412658 & ZNF676 & $C / T$ & 0.35 & $\mathrm{~T}$ & 1.01 & $0.91-1.12$ & 0.866 & 1.02 & $0.88-1.18$ & 0.826 & 1.01 & $0.81-1.27$ & 0.920 \\
\hline rs6028466 & DHX35 & $\mathrm{G} / \mathrm{A}$ & 0.07 & A & 1.23 & $1.08-1.50$ & 0.042 & 1.25 & $1.00-1.55$ & 0.048 & 1.33 & $0.55-3.15$ & 0.505 \\
\hline rs755017 & ZBTB46 & $\mathrm{A} / \mathrm{G}$ & 0.12 & G & 1.10 & $0.93-1.29$ & 0.235 & 1.05 & $0.88-1.25$ & 0.609 & 1.61 & $0.88-2.93$ & 0.120 \\
\hline
\end{tabular}

${ }^{a} M A F$ minor allele frequency, EA effect allele, allele associated with longer telomere length, OR odds ratio, $95 \% \mathrm{Cl} 95 \%$ coefficient interval.

${ }^{\mathrm{b}}$ Allelic model: $\mathrm{M}$ vs. $\mathrm{m}$, common allele vs. rare allele.

'Codominant model: Mm vs. MM, heterozygous carriers vs. common homozygous, mm vs. MM, rare homozygous vs. common homozygous. 
Table 3 Association between teloscore and MM risk.

\begin{tabular}{|c|c|c|c|c|c|c|c|}
\hline Type of score & Quintiles & Controls & Cases & Total & OR & $95 \% \mathrm{Cl}$ & $P_{\text {value }}$ \\
\hline \multirow[t]{6}{*}{ Unweighted, subjects with $100 \%$ call rate } & 1 & 342 & 438 & 780 & 1.00 & - & Ref. \\
\hline & 2 & 250 & 344 & 594 & 1.03 & $0.81-1.31$ & 0.788 \\
\hline & 3 & 226 & 327 & 553 & 1.18 & $0.93-1.51$ & 0.178 \\
\hline & 4 & 180 & 293 & 473 & 1.32 & $1.02-1.71$ & 0.032 \\
\hline & 5 & 209 & 367 & 576 & 1.36 & $1.07-1.73$ & 0.013 \\
\hline & Continuous $^{\mathrm{a}}$ & 1207 & 1769 & 2976 & 1.09 & $1.03-1.15$ & $2.53 \times 10^{-3}$ \\
\hline \multirow[t]{6}{*}{ Unweighted scaled, all subjects } & 1 & 478 & 522 & 1000 & 1.00 & - & Ref. \\
\hline & 2 & 356 & 449 & 805 & 1.09 & $0.88-1.34$ & 0.422 \\
\hline & 3 & 338 & 448 & 786 & 1.25 & $1.01-1.55$ & 0.037 \\
\hline & 4 & 252 & 440 & 692 & 1.59 & $1.28-1.99$ & $4.17 \times 10^{-5}$ \\
\hline & 5 & 317 & 548 & 865 & 1.58 & $1.28-1.94$ & $1.93 \times 10^{-5}$ \\
\hline & Continuous $^{\mathrm{a}}$ & 1741 & 2407 & 4148 & 1.14 & $1.08-1.19$ & $1.89 \times 10^{-7}$ \\
\hline \multirow[t]{6}{*}{ Weighted, subjects with $100 \%$ call rate } & 1 & 244 & 293 & 537 & 1.00 & - & Ref. \\
\hline & 2 & 248 & 315 & 563 & 1.02 & $0.78-1.32$ & 0.905 \\
\hline & 3 & 234 & 363 & 597 & 1.26 & $0.96-1.63$ & 0.085 \\
\hline & 4 & 241 & 387 & 628 & 1.35 & $1.04-1.75$ & 0.022 \\
\hline & 5 & 240 & 411 & 651 & 1.39 & $1.08-1.80$ & 0.011 \\
\hline & Continuous $^{\mathrm{a}}$ & 1207 & 1769 & 2976 & 1.10 & $1.04-1.16$ & $1.20 \times 10^{-3}$ \\
\hline \multirow[t]{6}{*}{ Weighted scaled, all subjects } & 1 & 356 & 361 & 717 & 1.00 & - & Ref. \\
\hline & 2 & 342 & 386 & 728 & 1.01 & $0.79-1.27$ & 0.940 \\
\hline & 3 & 348 & 493 & 841 & 1.29 & $1.03-1.62$ & 0.026 \\
\hline & 4 & 348 & 546 & 894 & 1.49 & $1.19-1.86$ & $4.80 \times 10^{-4}$ \\
\hline & 5 & 347 & 621 & 968 & 1.69 & $1.36-2.11$ & $2.97 \times 10^{-6}$ \\
\hline & Continuous $^{\mathrm{a}}$ & 1741 & 2407 & 4148 & 1.15 & $1.09-1.21$ & $1.07 \times 10^{-8}$ \\
\hline
\end{tabular}

${ }^{\mathrm{a}}$ The estimate measures the increase in risk associated with each increase of one quintile.

approach as previously described. The IVW regression showed a highly significant association between the 11 SNPs used as genetic instruments of exposure and risk of developing MM (coeff $=1.175$, 95\% CI $0.61-1.75, P=5.38 \times$ $10^{-5}$ ), whereas the MR-Egger regression showed a statistically non-significant association (coeff $=0.532,95 \% \mathrm{CI}$ -1.16 to $2.22, P=0.538$ ), although in line with IVW regression, with the intercept slightly, but not significantly, different from zero (intercept $=0.06,95 \% \mathrm{CI}-0.09$ to 0.20 , $P=0.428$ ). The $\mathrm{Q}^{\prime}$ heterogeneity statistic did not show statistically significant heterogeneity between the polymorphisms $(P=0.111)$, and Bowden $\mathrm{I}_{\mathrm{GX}}^{2}$ statistic showed that the selected genetic variants have high power to detect directional pleiotropy and causal effect for MR-Egger $\left(I^{2}=\right.$ 93.1\%). Scatter plots and forest plot of the causal estimate for risk of developing MM are shown in Supplementary Figs. 1 and 2, respectively.

\section{Association between individual SNPs and teloscore with MM OS}

Survival analysis did not show any statistically significant associations if considering multiple testing. However, we found an association between TERCrs10936599-T allele carriers and worse MM survival compared to $\mathrm{C}$ allele carriers $\left(\mathrm{HR}_{\text {allelic }}=1.20 ; 95 \% \mathrm{CI}\right.$ $1.00-1.43 ; P=0.048)$ and an association between A allele homozygous of the PXK-rs6772228 SNP and worse survival compared to homozygous for the common $\mathrm{T}$ allele $\left(\mathrm{HR}_{\text {homozygous }}=4.51 ; \quad 95 \% \quad \mathrm{CI} \quad 1.06-19.17 ; \quad P=0.041\right)$ (Table 4 and Supplementary Table 4).

The analyses between teloscore and OS of MM patients showed a trend between having longer gdTL and better MM survival, considering the quintile as a continuous variable for unweighted $(\mathrm{HR}=0.93$; 95\%CI $0.85-0.99$; $P=0.046)$ or weighted $(\mathrm{HR}=0.93 ; 95 \%$ CI $0.86-0.99$; 
Table 4 Association between individual SNPs and MM OS, adjusted by stage (Durie-Salmon).

\begin{tabular}{|c|c|c|c|c|c|c|c|c|c|c|c|c|c|}
\hline \multirow[t]{2}{*}{ SNP } & \multirow[t]{2}{*}{ Gene } & \multirow{2}{*}{$\begin{array}{l}\text { Allele } \\
\mathrm{M} / \mathrm{m}\end{array}$} & \multirow[t]{2}{*}{$\mathrm{MAF}^{\mathrm{a}}$} & \multirow[t]{2}{*}{$E A^{a}$} & \multicolumn{3}{|c|}{ Allelic model $^{\mathbf{b}}$} & \multicolumn{6}{|c|}{ Codominant model $^{\mathrm{C}}$} \\
\hline & & & & & $H^{a}$ & $95 \% \mathrm{Cl}^{\mathrm{a}}$ & $P_{\text {value }}$ & $\mathrm{HR}_{\text {Het }}$ & $95 \% \mathrm{Cl}$ & $P_{\text {value }}$ & $\mathrm{HR}_{\text {Hom }}$ & $95 \% \mathrm{Cl}$ & $P_{\text {value }}$ \\
\hline rs11125529 & ACYP2 & $C / A$ & 0.11 & A & 0.90 & $0.71-1.13$ & 0.369 & 0.89 & $0.68-1.16$ & 0.391 & 0.59 & $0.37-1.93$ & 0.710 \\
\hline rs6772228 & PXK & T/A & 0.04 & $\mathrm{~T}$ & 1.30 & $0.89-1.89$ & 0.165 & 1.18 & $0.78-1.75$ & 0.426 & 4.51 & $1.06-19.17$ & 0.041 \\
\hline rs10936599 & TERC & $C / T$ & 0.24 & C & 1.20 & $1.00-1.43$ & 0.048 & 1.23 & $0.98-1.54$ & 0.066 & 1.34 & $0.80-2.23$ & 0.265 \\
\hline rs7675998 & NAF1 & $\mathrm{G} / \mathrm{A}$ & 0.24 & G & 1.18 & $0.98-1.42$ & 0.080 & 1.11 & $0.88-1.40$ & 0.374 & 1.62 & $0.99-2.63$ & 0.053 \\
\hline rs2736100 & TERT & $\mathrm{A} / \mathrm{C}$ & 0.50 & C & 1.05 & $0.89-1.22$ & 0.536 & 1.02 & $0.77-1.34$ & 0.879 & 1.10 & $0.70-1.49$ & 0.545 \\
\hline rs9420907 & OBFC1 & $\mathrm{A} / \mathrm{C}$ & 0.13 & C & 0.96 & $0.78-1.17$ & 0.724 & 0.95 & $0.75-1.20$ & 0.690 & 0.95 & $0.51-1.85$ & 0.941 \\
\hline rs3027234 & CTC1 & $C / T$ & 0.22 & $C$ & 1.03 & $0.86-1.23$ & 0.726 & 1.00 & $0.79-1.25$ & 0.993 & 1.15 & $0.71-1.83$ & 0.559 \\
\hline rs8105767 & ZNF208 & $A / G$ & 0.28 & G & 1.01 & $0.85-1.19$ & 0.913 & 1.06 & $0.84-1.32$ & 0.612 & 0.95 & $0.62-1.42$ & 0.799 \\
\hline rs412658 & ZNF676 & $C / T$ & 0.35 & T & 0.98 & $0.83-1.15$ & 0.847 & 1.01 & $0.80-0.25$ & 0.943 & 0.95 & $0.66-1.35$ & 0.763 \\
\hline rs6028466 & DHX35 & $\mathrm{G} / \mathrm{A}$ & 0.07 & A & 1.15 & $0.86-1.52$ & 0.350 & 1.10 & $0.78-1.54$ & 0.562 & 1.58 & $0.58-4.27$ & 0.369 \\
\hline rs755017 & ZBTB46 & $A / G$ & 0.12 & G & 0.86 & $0.67-1.09$ & 0.216 & 0.92 & $0.70-1.20$ & 0.559 & 0.44 & $0.14-1.37$ & 0.158 \\
\hline
\end{tabular}

${ }^{a} M A F$ minor allele frequency, EA effect allele, allele associated with longer telomere length, $H R$ hazard ratio, $95 \% \mathrm{Cl} 95 \%$ coefficient interval.

${ }^{b}$ Allelic model: $M$ vs. $\mathrm{m}$, common allele vs. rare allele.

'Codominant model: Mm vs. MM, heterozygous carriers vs. common homozygous; mm vs. MM, rare homozygous vs. common homozygous.

$P=0.049)$ score, adjusted by DS staging system. Similar results were obtained in the analyses adjusted by ISS (Table 5).

The results of the Mendelian randomization analysis performed with IVW regression and MR-Egger regression showed an inverse, non-significant association between MM survival and the genetic score built with the 11 SNPs (coeff $=-0.44,95 \% \mathrm{CI}-1.18$ to $0.29, P=0.238$ ), with the intercept of the MR-Egger regression non-significantly different from zero (coeff $=-0.108,95 \% \mathrm{CI}-0.28$ to 0.07 , $P=0.227)$. $\mathrm{Q}^{\prime}$ heterogeneity $(P=0.391)$ did not show statistically significant heterogeneity between the polymorphisms, and Bowden $\mathrm{I}_{\mathrm{GX}}^{2}$ statistic did not show weak instrument bias (92.8\%). Scatter plots and forest plot of the causal estimate for risk of developing MM are shown in Supplementary Figs. 3 and 4, respectively.

\section{In silico identification of functional effects}

We tested the possible functional relevance of CTC1rs3027234, ZNF208-rs8105767, OBFC1-rs9420907, TERC -rs10936599, and $P X K$-rs6772228, that were significant in at least one analysis and the variants in linkage disequilibrium (LD) with them using RegulomeDB and GTEx.

RegulomeDB showed a value of $1 \mathrm{~b}$ for CTC1-rs3027234 that indicates the presence of an eQTL, of at least one transcription factor binding site and the region to be sensitive to the action of DNase. Analyzing rs3027234 in the GTEx portal, a correlation between the $\mathrm{T}$ allele and lower gene expression was observed in 20 tissues including whole blood, where it showed a strongly significant association $\left(P=1.2 \times 10^{-33}\right)$. RegulomeDB assigned to ZNF208-rs8105767 a value of $1 \mathrm{f}$, which indicates a functional role similar to CTC1-rs3027234. GTEx reported six associations with different genes for rs8105767, and the strongest correlation was between the A allele and lower expression of the ZNF257 gene $(P=$ $1.6 \times 10^{-25}$ ) in whole blood. RegulomeDB assigned to OBFC1-rs9420907 and TERC-rs10936599 a value of 3a, indicating the presence of at least one transcription factor binding site and the region to be sensitive to the action of DNase, and GTEx did not show any eQTL. RegulomeDB assigned to $P X K$-rs6772228 a value of 7 , indicating the absence of a functional role. Despite that, rs6772228 is in LD with rs77480019 $\left(r^{2}=0.92\right)$ and $\operatorname{rs} 73077957\left(r^{2}=\right.$ 0.84 ) for which RegulomeDB assigned a value of $3 \mathrm{a}$, but GTEx did not show any eQTL in whole blood.

\section{Discussion}

Telomere length is considered a risk factor, or a risk marker, for a large number of diseases including several cancer types ${ }^{18-25}$. However, in spite of the number of studies, including several with considerable sample size, it is still unclear whether long or short telomeres are responsible for the increase in risk. In addition to true biological differences in various diseases, a possible explanation for this uncertainty may be explained by the technical heterogeneity due to sample handling and study design used across the studies. For MM only two relatively small studies have been attempted, one in a retrospective case-control setting ${ }^{55}$ and the other in a prospective cohort $^{26}$, both reporting an association between longer 
Table 5 Association between teloscore and MM OS.

\begin{tabular}{|c|c|c|c|c|c|c|c|c|c|c|c|c|c|}
\hline \multirow[t]{2}{*}{ Type of score } & \multirow[t]{2}{*}{ Quintiles } & \multicolumn{6}{|c|}{ OS adjusted by stage (DS) } & \multicolumn{6}{|c|}{ OS adjusted by stage (ISS) } \\
\hline & & Alive & Deceased & Total & $\mathrm{HR}$ & $95 \% \mathrm{Cl}$ & $P_{\text {value }}$ & Alive & Deceased & Total & HR & $95 \% \mathrm{Cl}$ & $P_{\text {value }}$ \\
\hline \multirow{6}{*}{$\begin{array}{l}\text { Unweighted, subjects with } \\
100 \% \text { call rate }\end{array}$} & 1 & 158 & 65 & 223 & 1.00 & - & Ref. & 130 & 45 & 175 & 1.00 & - & Ref. \\
\hline & 2 & 142 & 52 & 194 & 1.11 & $0.77-1.60$ & 0.564 & 115 & 41 & 156 & 1.21 & $0.78-1.85$ & 0.382 \\
\hline & 3 & 134 & 48 & 182 & 0.98 & $0.67-1.43$ & 0.930 & 120 & 36 & 156 & 0.92 & $0.59-1.43$ & 0.726 \\
\hline & 4 & 117 & 42 & 159 & 0.72 & $0.48-1.07$ & 0.106 & 164 & 50 & 214 & 0.77 & $0.51-1.15$ & 0.210 \\
\hline & 5 & 133 & 43 & 176 & 0.84 & $0.57-1.24$ & 0.393 & 54 & 17 & 71 & 0.89 & $0.50-1.56$ & 0.685 \\
\hline & Continuous $^{\mathrm{a}}$ & 684 & 250 & 934 & 0.93 & $0.85-1.01$ & 0.103 & 583 & 189 & 772 & 0.92 & $0.82-1.02$ & 0.138 \\
\hline \multirow{6}{*}{$\begin{array}{l}\text { Unweighted scaled, all } \\
\text { subjects }\end{array}$} & 1 & 182 & 92 & 274 & 1.00 & - & Ref. & 152 & 71 & 223 & 1.00 & - & Ref. \\
\hline & 2 & 174 & 77 & 251 & 1.06 & $0.78-1.44$ & 0.684 & 139 & 56 & 195 & 1.07 & $0.74-1.52$ & 0.716 \\
\hline & 3 & 172 & 65 & 237 & 0.90 & $0.65-1.24$ & 0.538 & 154 & 50 & 204 & 0.83 & $0.57-1.19$ & 0.317 \\
\hline & 4 & 233 & 94 & 327 & 0.84 & $0.63-1.12$ & 0.251 & 196 & 69 & 265 & 0.77 & $0.55-1.08$ & 0.136 \\
\hline & 5 & 109 & 37 & 146 & 0.73 & $0.49-1.06$ & 0.106 & 102 & 33 & 135 & 0.73 & $0.48-1.12$ & 0.154 \\
\hline & Continuous $^{\mathrm{a}}$ & 870 & 365 & 1235 & 0.93 & $0.85-0.99$ & 0.046 & 743 & 279 & 1022 & 0.91 & $0.83-0.99$ & 0.037 \\
\hline \multirow{6}{*}{$\begin{array}{l}\text { Weighted, subjects with } \\
100 \% \text { call rate }\end{array}$} & 1 & 136 & 54 & 190 & 1.00 & - & Ref. & 119 & 37 & 156 & 1.00 & - & Ref. \\
\hline & 2 & 141 & 54 & 195 & 1.06 & $0.72-1.54$ & 0.765 & 111 & 48 & 159 & 1.27 & $0.82-1.96$ & 0.268 \\
\hline & 3 & 127 & 49 & 176 & 1.02 & $0.69-1.50$ & 0.917 & 116 & 36 & 152 & 0.94 & $0.59-1.49$ & 0.797 \\
\hline & 4 & 144 & 44 & 188 & 0.72 & $0.48-1.07$ & 0.108 & 121 & 30 & 151 & 0.66 & $0.40-1.07$ & 0.094 \\
\hline & 5 & 136 & 49 & 185 & 0.91 & $0.61-1.34$ & 0.626 & 116 & 38 & 154 & 1.01 & $0.64-1.60$ & 0.933 \\
\hline & Continuous $^{\mathrm{a}}$ & 684 & 250 & 934 & 0.94 & $0.86-1.03$ & 0.198 & 583 & 189 & 772 & 0.94 & $0.84-1.04$ & 0.225 \\
\hline \multirow{6}{*}{$\begin{array}{l}\text { Weighted scaled, all } \\
\text { subjects }\end{array}$} & 1 & 168 & 83 & 251 & 1.00 & - & Ref. & 147 & 64 & 211 & 1.00 & - & Ref. \\
\hline & 2 & 167 & 78 & 245 & 1.06 & $0.77-1.44$ & 0.722 & 138 & 66 & 204 & 1.06 & $0.75-1.50$ & 0.726 \\
\hline & 3 & 173 & 73 & 246 & 0.97 & $0.70-1.32$ & 0.837 & 148 & 51 & 199 & 0.86 & $0.59-1.24$ & 0.426 \\
\hline & 4 & 181 & 66 & 247 & 0.76 & $0.55-1.05$ & 0.107 & 158 & 46 & 204 & 0.67 & $0.45-0.98$ & 0.042 \\
\hline & 5 & 181 & 65 & 246 & 0.81 & $0.58-1.12$ & 0.210 & 152 & 52 & 204 & 0.82 & $0.57-1.19$ & 0.310 \\
\hline & Continuous $^{\mathrm{a}}$ & 870 & 365 & 1235 & 0.93 & $0.86-0.99$ & 0.049 & 743 & 279 & 1022 & 0.92 & $0.84-0.99$ & 0.046 \\
\hline
\end{tabular}

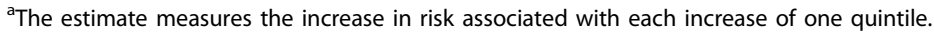

telomeres and increased risk of developing the disease. Telomere length is at least in part genetically determined and there are several studies that have successfully used SNPs as genetic surrogates for telomere length to infer risk of developing several tumor types ${ }^{23,37-45}$. This approach has never been attempted in MM. LTL has been tested in relation to cancer survival ${ }^{27}$, but never in $\mathrm{MM}$ and the only study conducted in MM is very small $(n=5)$ and used purified bone marrow cells and not whole blood $^{28}$.

Considering that LTL measure could be influenced by several epidemiologic confounders or methodological issues, the main advantage of the strategy used in this study is that the exposure (the genotypes) is not influenced by study design (prospective vs. retrospective), by sample manipulation or by environmental variables since genotypes are invariable throughout life. The aim of this study was to test whether GWAS-identified SNPs involved in telomere length, combined in a score, were associated with MM risk and, for the first time, OS of MM patients. Considering that individually each of the SNPs explains a small proportion of LTL variability, we have combined them in a score and performed a MR analysis to better characterize the relation between gdTL and the disease risk and survival.

We found a novel association between carriers of the $C$ allele OBFC1-rs9420907 SNP and increased MM risk $(P=$ $\left.7.78 \times 10^{-4}\right)$. The $\mathrm{C}$ allele of the SNP is also associated with longer telomeres ${ }^{34,38}$. This gene product is associated to the shelterin complex and is also a subunit of alpha accessory factor (AAF) that is involved in the initiation of DNA replication. We observed an increase in risk 
associated with the $\mathrm{C}$ allele of the rs3027234 polymorphisms and with $\mathrm{G}$ allele of the rs8105767. The CTC1rs3027234-C allele is associated with longer telomeres and, according to GTEx, with an increased expression of the gene in blood with a very high statistical significance $(p=$ $\left.1.2 \times 10^{-33}\right)$. It is therefore plausible that the $\mathrm{C}$ allele contributes to an increase efficiency of the complex that in turn results in longer telomeres that increase the risk of developing MM. The G allele of rs8105767 is associated with longer telomeres ${ }^{34}$ and, according to GTEX, it has an effect on the expression of various genes in whole blood, mainly ZNF257 $\left(P=1.6 \times 10^{-25}\right)$.

The main finding of this manuscript is the observation that gdTL is associated with increased risk of MM. This association is consistent in all the models we tested and when using all the subjects it reached a very high statistical significance, especially comparing individuals with the longest telomeres with individuals with the shortest telomeres (quintile 5 vs. quintile $1 ; P=2.97 \times 10^{-6}$ ) or when considering the quintile variable as continuous $(P=1.07 \times$ $10^{-8}$ ). This finding is in agreement with what was found in the two studies that reported longer telomeres measured by real-time PCR to be associated with increased risk of developing the disease $\mathrm{e}^{26,55}$ and it is also in line with a very large study performed on a substantial number of different cancer types, where the general trend for most cancers was an association between longer genetically determined telomeres and increased risk ${ }^{23}$. To analyze in depth the association between gdTL and risk of MM, the 11 SNPs selected were analyzed with a Mendelian randomization approach to test the presence of directional pleiotropy and its influence in the estimate of the causal effect between LTL and risk of developing MM. The results were in line with the finding obtained through teloscore analysis, supporting the evidence for a causal effect of longer gdTL with an increased MM risk. Moreover, the MR-Egger intercept was not statistically different from zero and the results of the heterogeneity tests were not statistically significant. These observations suggest the absence of directional pleiotropy and heterogeneity between the polymorphisms selected.

From a biologic point of view, longer telomeres (particularly as measured by the SNP teloscore, which represents the innate tendency to have longer or shorter telomeres) could represent a marker of a cell that has a higher dividing potential and therefore an increased tendency in acquiring new potential harmful mutations ${ }^{23}$.

A potential limitation is that most of the controls used in this study, as for almost all case-control studies, are either blood donors or hospitalized individuals with pathologies unrelated to MM. Considering that gdLTL has been associated with several human traits, this may represent a confounding factor of the analysis.

A point of novelty is represented by two borderline associations between the allele associated with shorter TL and a worse OS of MM patients, compared to the allele associated with longer TL, both for TERC-rs10936599 $(P=0.048)$ and $P X K-6772228(P=0.041)$. Furthermore, the teloscore survival analyses showed that longer gdTL is correlated with better MM patients OS compared to patients with shorter gdTL. Despite this, the different direction obtained for IVW and MR-Egger analyses did not support a significant association of the teloscore with OS of MM patients, although the Cochran's Q statistic did not show evidence of heterogeneity between the polymorphisms. A possible explanation for Mendelian randomization results is that the increase of patient's survival could be due to pleiotropic effects of the genetic variants that act on the outcome via a confounder.

$\mathrm{Xu}$ and colleagues conducted a thorough meta-analysis to test the effect of LTL in cancer progression and survival and even though MM was not included, their findings are in agreement with ours i.e., shorter telomere associated with worse prognosis ${ }^{27}$.

We do not have data for myeloma-specific mortality. On the other hand, although in the last years many advances in therapy have been made, MM remains an incurable disease and therefore we presume that the vast majority of deaths are disease specific.

It is worth noting that gdTL as measured in our work, being based on germline polymorphisms that do not change throughout life, is unaffected not only by age, but also by any other biological mechanism induced by environmental exposures (e.g., smoking) or therapeutic exposures (e.g., anticancer treatments) that are known to affect telomere length.

In conclusion, we present here convincing evidence that longer gdTL is a risk marker for MM development.

\footnotetext{
Acknowledgements

This work was partially supported by intramural funds of Univerity of Pisa and DKFZ; by Fondo de Investigaciones Sanitarias (Madrid, Spain) [PI12/02688 to J. S., PI17/02276 to J.S.]; by Instituto de Salud Carlos III, co-funded by FEDER funds — a way to build Europe-[P|14-00613 to V.M.] and by Agency for

Management of University and Research Grants (AGAUR) of the Catalan Government (Barcelona, Spain) [2017SGR723 to V.M.]. Open Access funding enabled and organized by Projekt DEAL.
}

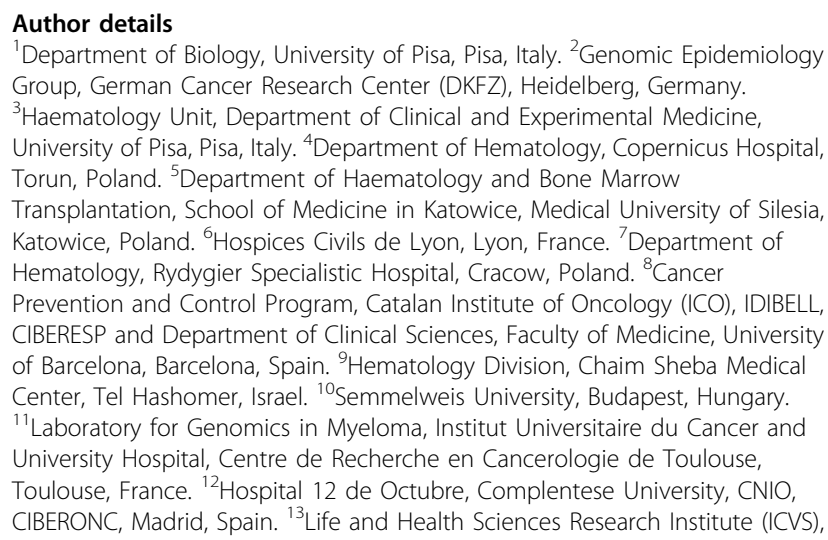


School of Health Sciences, University of Minho, Braga, Portugal and ICVS/3B's PT Government Associate Laboratory, Braga/Guimarães, Portugal.

${ }^{14}$ Department of Hematology, Holy Cross Cancer Center, Kielce, Poland. ${ }^{15}$ Department of Hematology, Institute of Hematology and Transfusion Medicine, Warsaw, Poland. ${ }^{16}$ Hematology Department, University Hospital of Salamanca, CIBERONC, Salamanca, Spain. ${ }^{17}$ Department of Biochemistry, University Hospital of Southern Jutland, Sønderborg, Denmark. ${ }^{18}$ IRS-Center Soenderjylland, University Hospital of Southern Jutland, Aabenraa, Denmark. ${ }^{19}$ Department of Hematology, Jagiellonian University Medical College, Krakow, Poland. ${ }^{20}$ Department of Hematology, University Hospital No. 2 in Bydgoszcz, Bydgoszcz, Poland. ${ }^{21}$ Department of Hematology, Odense University Hospital, Odense, Denmark. ${ }^{22}$ Department of Hematology, Institute of Medical Sciences, College of Medical Sciences, University of Rzeszow, Rzeszow, Poland.

${ }^{23}$ Department of Internal Diseases, Occupational Medicine, Hypertension and Clinical Oncology, Wroclaw Medical University, Wroclaw, Poland. ${ }^{24}$ Department of Hematology, Rigshospitalet, Copenhagen, Denmark. ${ }^{25}$ Department of Haemato-oncology and Bone Marrow Transplantation and Department of Internal Medicine in Nursing, Medical University of Lublin, Lublin, Poland. ${ }^{26}$ Department of Hematology, Aarhus University Hospital, Aarhus, Denmark. ${ }^{27}$ Department of Hematology and Transplantology Medical University of Gdansk, Gdańsk, Poland. ${ }^{28}$ Inserm, U900, Institut Curie, PSL University, Mines ParisTech, Paris, France. ${ }^{29}$ U.O. Dipartimento di Ematologia, Azienda USL Toscana Nord Ovest, Livorno, Italy, currently Ospedale Santa Chiara, Pisa, Italy. ${ }^{30}$ Molecular Oncology Research Center, Barretos Cancer Hospital, Barretos, Brazil. ${ }^{31}$ Department of Hematology, Medical University of Lodz, Łódź, Poland. ${ }^{32}$ Department of Hematology, Military Institute of Medicine, Warsaw, Poland. ${ }^{33}$ Department of Haematology, University Hospital in Cracow, Cracow, Poland. ${ }^{34}$ Department of Lymphoid Malignancies, Maria Skłodowska-Curie National Research Institute of Oncology, Warsaw, Poland. ${ }^{35}$ Laboratory of Clinical and Transplant Immunology and Genetics, Copernicus Memorial Hospital, Łódź, Poland. ${ }^{36}$ Genomic Oncology Area, GENYO, Centre for Genomics and Oncological Research: Pfizer/University of Granada/Andalusian Regional Government, Granada, Spain. ${ }^{37}$ Hematology Department, Virgen de las Nieves University Hospital, Granada, Spain

\section{Conflict of interest}

The authors declare no competing interests.

\section{Publisher's note}

Springer Nature remains neutral with regard to jurisdictional claims in published maps and institutional affiliations.

Supplementary information The online version contains supplementary material available at https://doi.org/10.1038/s41408-021-00462-y.

Received: 7 September 2020 Revised: 1 March 2021 Accepted: 11 March 2021

Published online: 14 April 2021

\section{References}

1. Kumar, S. K. et al. Multiple myeloma. Nat. Rev. Dis. Prim. 3, 17046 (2017).

2. Broderick, P. et al. Common variation at 3p22.1 and 7p15.3 influences multiple myeloma risk. Nat. Genet. 44, 58-61 (2011).

3. Campa, D. et al. Comprehensive investigation of genetic variation in the $8 \mathrm{q} 24$ region and multiple myeloma risk in the IMMEnSE consortium. Br. J. Haematol. 157, 331-338 (2012).

4. Chubb, D. et al. Common variation at 3q26.2, 6p21.33, 17p11.2 and 22q13.1 influences multiple myeloma risk. Nat. Genet. 45, 1221-1225 (2013).

5. Martino, A. et al. Polymorphisms in xenobiotic transporters ABCB1, ABCG2, $A B C C 2, A B C C 1, A B C C 3$ and multiple myeloma risk: a case-control study in the context of the International Multiple Myeloma rESEarch (IMMEnSE) consortium. Leukemia. 26, 1419-1422 (2012).

6. Martino, A. et al. Impact of polymorphic variation at 7p15.3, 3p22.1 and 2 p23.3 loci on risk of multiple myeloma. Br. J. Haematol. 158, 805-809 (2012).

7. Vangsted, A., Klausen, T. W. \& Vogel, U. Genetic variations in multiple myeloma I: effect on risk of multiple myeloma. Eur. J. Haematol. 88, 8-30 (2012).
8. Weinhold, $\mathrm{N}$. et al. The CCND1 c.870G $>$ A polymorphism is a risk factor for $t$ (11;14)(q13;q32) multiple myeloma. Nat. Genet. 45, 522-525 (2013).

9. Ziv, E. et al. Genome-wide association study identifies variants at $16 \mathrm{p} 13$ associated with survival in multiple myeloma patients. Nat. Commun. 6, 7539 (2015).

10. Johnson, D. C. et al. Genome-wide association study identifies variation at 6q25.1 associated with survival in multiple myeloma. Nat. Commun. 7, 10290 (2016).

11. Erickson, S. W. et al. A common genetic variant in 19q13.3 is associated with outcome of multiple myeloma patients treated with Total Therapy 2 and 3. Br. J. Haematol. 174, 991-993 (2016).

12. Ríos-Tamayo, R. et al. A common variant within the HNF1B gene is associated with overall survival of multiple myeloma patients: results from the IMMEnSE consortium and meta-analysis. Oncotarget 7, 59029-59048 (2016).

13. Macauda, A. et al. Inherited variation in the xenobiotic transporter pathway and survival of multiple myeloma patients. Br. J. Haematol. 183, 375-384 (2018).

14. Łacina, P., Butrym, A., Mazur, G. \& Bogunia-Kubik K. BSG and MCT1 genetic variants influence survival in multiple myeloma patients. Genes. 9, 226 (2018).

15. Campa, D. et al. Genetic polymorphisms in genes of class switch recombination and multiple myeloma risk and survival: an IMMEnSE study. Leuk Lymphoma. 60, 1803-1811 (2019).

16. de Lange, T. How telomeres solve the end-protection problem. Science. 326, 948-952 (2009)

17. Blasco, M. A. Telomeres and human disease: ageing, cancer and beyond. Nat. Rev. Genet. 6, 611-622 (2005).

18. Campa, D. et al. Mitochondrial DNA copy number variation, leukocyte telomere length, and breast cancer risk in the European Prospective Investigation into Cancer and Nutrition (EPIC) study. Breast Cancer Res. 20, 29 (2018).

19. Wentzensen, I. M., Mirabello, L., Pfeiffer, R. M. \& Savage, S. A. The association of telomere length and cancer: a meta-analysis. Cancer Epidemiol. Biomarkers Prev. 20, 1238-1250 (2011).

20. $\mathrm{Ma}, \mathrm{H}$. et al. Shortened telomere length is associated with increased risk of cancer: a meta-analysis. PLoS ONE 6, e20466 (2011).

21. Campa, D. et al. Risk of multiple myeloma is associated with polymorphisms within telomerase genes and telomere length. Int. J. Cancer 136, E351-E358 (2015).

22. Campa, D. et al. Leukocyte telomere length in relation to pancreatic cancer risk: a prospective study. Cancer Epidemiol. Biomarkers Prev. 23, 2447-2454 (2014).

23. Haycock, P. C. et al. Association between telomere length and risk of cancer and non-neoplastic diseases. JAMA Oncol. 3, 636 (2017).

24. Wang, S. et al. Association between leukocyte telomere length and glioma risk: a case-control study. Neuro. Oncol. 16, 505-512 (2014).

25. Weischer, M. et al. Short telomere length, cancer survival, and cancer risk in 47102 individuals. J. Natl Cancer Inst. 105, 459-468 (2013).

26. Hosnijeh, F. S. et al. Prediagnostic telomere length and risk of B-cell lymphoma-Results from the EPIC cohort study. Int. J. Cancer 135, 2910-2917 (2014).

27. $\mathrm{Xu}, \mathrm{X}$. et al. Association between telomere length and survival in cancer patients: a meta-analysis and review of literature. Front. Med. 10, 191-203 (2016).

28. Hyatt, S. et al. Telomere length is a critical determinant for survival in multiple myeloma. Br. J. Haematol. 178, 94-98 (2017).

29. Kimura, M. et al. Synchrony of telomere length among hematopoietic cells. Exp. Hematol. 38, 854-859 (2010).

30. Wilson, W. R. W. et al. Blood leucocyte telomere DNA content predicts vascular telomere DNA content in humans with and without vascular disease. Eur. Heart J. 29, 2689-2694 (2008).

31. Barrett, J. H., lles, M. M., Dunning, A. M. \& Pooley, K. A. Telomere length and common disease: study design and analytical challenges. Hum. Genet. 134, 679-689 (2015).

32. Coutts, F. et al. The polygenic nature of telomere length and the anti-ageing properties of lithium. Neuropsychopharmacology. 44, 757-765 (2019).

33. Kim, J.H. et al. Heritability of telomere length across three generations of Korean families. Pediatr. Res. 87, 1060-1065 (2020).

34. Codd, V. et al. Identification of seven loci affecting mean telomere length and their association with disease. Nat. Genet. 45, 422-427 (2013).

35. Mangino, M. et al. Genome-wide meta-analysis points to CTC1 and ZNF676 as genes regulating telomere homeostasis in humans. Hum. Mol. Genet. 21, 5385-5394 (2012). 
36. Levy, D. et al. Genome-wide association identifies OBFC1 as a locus involved in human leukocyte telomere biology. Proc. Natl Acad. Sci. USA 107, 9293-9298 (2010).

37. Campa, D. et al. Genetic determinants of telomere length and risk of pancreatic cancer: a PANDoRA study. Int. J. Cancer 144, 1275-1283 (2019).

38. Iles, M. M. et al. The effect on melanoma risk of genes previously associated with telomere length. J. Natl Cancer Inst. 106, dju267 (2014).

39. Machiela, M. J. et al. Genetic variants related to longer telomere length are associated with increased risk of renal cell carcinoma. Eur. Urol. 72, 747-754 (2017).

40. Machiela, M. J. et al. Genetically predicted longer telomere length is associated with increased risk of B-cell lymphoma subtypes. Hum. Mol. Genet. 25, 1663-1676 (2016)

41. Walsh, K. M. et al. Longer genotypically-estimated leukocyte telomere length is associated with increased adult glioma risk. Oncotarget. 6, 42468-42477 (2015).

42. Walsh, K. M. et al. Common genetic variants associated with telomere length confer risk for neuroblastoma and other childhood cancers. Carcinogenesis. 37, 576-582 (2016)

43. Zhang, C. et al. Genetic determinants of telomere length and risk of common cancers: a Mendelian randomization study. Hum. Mol. Genet. 24, 5356-5366 (2015).

44. Luu, H. N. et al. Association between genetic risk score for telomere length and risk of breast cancer. Cancer Causes Control 27, 1219-1228 (2016).

45. Giaccherini, M. et al. Genetic polymorphisms associated with telomere length and risk of developing myeloproliferative neoplasms. Blood Cancer J. 10, 89 (2020).
46. Martino, A. et al. Genetics and molecular epidemiology of multiple myeloma: the rationale for the IMMEnSE consortium (review). Int. J. Oncol. 40, 625-638 (2012).

47. Palumbo, A. \& Anderson, K. Multiple myeloma. N. Engl. J. Med. 364, 1046-1060 (2011).

48. Durie, B. G. M. \& Salmon, S. E. A clinical staging system for multiple myeloma correlation of measured myeloma cell mass with presenting clinical features, response to treatment, and survival. Cancer 36, 842-854 (1975).

49. Greipp, P. R. et al. International staging system for multiple myeloma. J. Clin Oncol. 23, 3412-3420 (2005).

50. Burgess, S., Butterworth, A. \& Thompson, S. G. Mendelian randomization analysis with multiple genetic variants using summarized data. Genet. Epidemiol. 37, 658-665 (2013).

51. Bowden, J., Davey Smith, G. \& Burgess, S. Mendelian randomization with invalid instruments: effect estimation and bias detection through Egger regression. Int. J. Epidemiol. 44, 512-525 (2015).

52. Bowden, J. et al. Assessing the suitability of summary data for two-sample Mendelian randomization analyses using MR-Egger regression: the role of the 12 statistic. Int. J. Epidemiol. 45, dyw220 (2016).

53. Boyle, A. P. et al. Annotation of functional variation in personal genomes using RegulomeDB. Genome Res. 22, 1790-1797 (2012).

54. Lonsdale, J. et al. The genotype-tissue expression (GTEx) project. Nat. Genet. 45 580-585 (2013).

55. Campa, D. et al. Risk of multiple myeloma is associated with polymorphisms within telomerase genes and telomere length. Int. J. Cancer 136, E351-E358 (2015). 